\title{
Combined ANFIS-Dijkstra's Model: A Dynamic Simulation for Suburban Public Transportation
}

\author{
Nazanin Pilevari ${ }^{1}$, Maryam Rahmaty ${ }^{2}$, Mohammad Shokouhifar ${ }^{3+}$ \\ ${ }^{1}$ Department of Industrial Management, West Tehran Branch, Islamic Azad \\ University, Tehran, Iran \\ ${ }^{2}$ Department of Management, Chalous Branch, Islamic Azad University, Chalous, \\ Iran \\ ${ }^{3}$ Department of Electrical and Computer Engineering, Shahid Beheshti University, \\ Tehran, Iran \\ ${ }^{+}$m_shokouhifar@sbu.ac.ir.
}

\begin{abstract}
This paper presents a dynamic simulation based on a combination of Adaptive Neuro-Fuzzy Inference System (ANFIS) and Dijkstra's algorithm (named ANFIS-Dijkstra's model), to design and simulate a dynamic suburban public transportation system in Iran. To evaluate the proposed suburban public transportation system, two simulation methods related to the current system and the proposed ANFIS-Dijkstra's model are designed and implemented. To make the simulator closer to the reality, a machine learning technique based on ANFIS is used for route traffic estimation, while and Fuzzy C-Means (FCM) clustering algorithm is utilized to determine the number of passengers. Then, Dijkstra's algorithm is applied for the optimal routing of passengers from the source to the destination. Finally, the results of the two simulation models are compared to justify the performance of the proposed ANFIS-Dijkstra's model. Based on the results, the rate of using bus compared to taxi increased by $8.2 \%$, the rate of using bus capacity increased by $12.66 \%$, and the total fare paid by passengers decreased.
\end{abstract}

Keywords: Suburban transportation, Dynamic systems, Dijkstra's algorithm, Fuzzy C-Means (FCM), Adaptive Neuro-Fuzzy Inference System (ANFIS). 


\section{Introduction}

Transportation systems are inseparable parts of the economy. The social and economic health of an area highly depends on the performance of its transportation system. The transportation system not only facilitates mobility, but also affects the growth patterns and level of economic activity in the long term through access to different areas (Li and DaCosta, 2013). Due to the importance of the transportation systems in economy, industry, politics, and even the military, many experts consider transportation as the basis of sustainable development and believe that the development becomes more inclusive when transportation is more efficient. In other words, any movement should have most efficiency in terms of cost-benefit and environmental friendliness. On the contrary, inefficiency of the transportation system may lead to serious environmental consequences such as air pollution, negative social and economic consequences and cause the inefficiency of performance in cities.

In recent years, the need for transportation has increased along with economic growth and development. Consequently, complications such as increased energy consumption and environmental pollution intensity occur with the expansion of transportation activities (Hutchison 2010). During the last forty years, the trend of transportation activities around the world indicates growth in the level of car dependence and change in social lifestyle, leading to increase in sensitivity to environmental impacts and its reflections in health. The importance of transportation planning has been highlighted because of the highly critical role of transportation in everyday life and the disability of transportation routes to accept any volume of traffic, as well as the need to prevent accidents. On the other hand, transportation planning is impossible without road traffic information. Transportation will not improve in developing countries under the current pattern of transportation and pressures due to the rapid economic growth. Thus, the increase of congestion, pollution, negative effects on health, and energy consumption are expected. Based on the World Health Organization (WHO) report in 2004, road accidents with more than two million deaths per year would be the fifth leading cause of death in 2030. In addition, International Energy Agency (IEA) estimated in 2010 that energy consumption in the transportation sector would increase up to $50 \%$ by 2030 and $80 \%$ by 2050 under the current conditions (Hidalgo and Huizenga 2013).

Significance of suburban transportation systems as the most important symbol and the most basic prerequisite for development in any country is obvious. Currently, some suburban public vehicles pass between cities with empty seats on a daily basis. Nevertheless, many travelers fail to receive tickets to their desired destination at the right time. As a result, they would pay high fares for taxis, despite their inner willingness. On the other hand, if such individuals want to divide their route into smaller routes, they are not sure if they can get a ticket later. 
Based on the above-mentioned issues, this paper aims to conduct the combined design and simulation of a dynamic suburban public transportation system in Iran using a combined technique based on Adaptive Neuro-Fuzzy Inference System (ANFIS) and Dijkstra's algorithm. In the proposed ANFIS-Dijkstra's model, a transportation system is presented to enable the passenger to choose the most optimal route for reaching his destination at any time. As a result, the passenger will use the offered routes to use a combination of vehicles if she/he fails to get a ticket of the desired travel style and she/he considers the cost of the trip as important. In this regard, the passenger will get ensured that she/he is offered the best route in terms of cost and time based on his circumstances. In addition, the passenger ensures reaching the destination by booking tickets to continue the route by different vehicles. Such an integrated system can change the public transportation system especially since the proposed model can be generalized in terms of different vehicles to the desired number.

Simulations of the proposed model have been performed by Simulink and MATLAB software. All possible details and events have been considered in this software to convince the managers of travel agencies about its benefits and reduce the risk percentage as much as possible. The value of the designed transportation system is not limited to optimizing fuel consumption and reducing passenger costs. However, it provides a healthier environment due to the maximum use of bus capacity. The raised issues can satisfy all of these components, if we consider the fare paid by the passenger, the passenger's confidence in preparing the ticket, and the optimal routing in terms of time among the concerns and components affecting passenger satisfaction. Considering the accuracy of the proposed model on the used dataset, it is not dependent on the obtained dataset, and can be applied to other datasets.

The remainder of this paper is organized as follows. Section 2 reviews the existing literature. Section 3 proposes the preliminaries of the ANFIS model to forecast the traffic volume, and the Dijkstra's algorithm. Section 4 presents the proposed combined ANFIS-Dijkstra's methodology. In Section 5, the results and analysis are discussed, and finally, the paper is concluded in Section 6.

\section{Literature Review}

As stated by O'Flaherty (2002), "Early manufactured roads were the stone-paved streets of Ur in the Middle East (ca 4000 BC), the corduroy-log paths near Glastonbury, England (ca 3300 BC), and brick paving in India (ca 3000 BC). The oldest extant wooden pathway in Europe, the $2 \mathrm{~km}$ long Sweet Track, was built across (parts subsequently preserved in) marshy ground near Glastonbury. The oldest extant stone road in Europe was built in Crete ca 2000 BC". Therefore, the need for transportation dates back to the history of civilization. The first means of transportation included passages built in the forests by opening the ways. The roads 
constructions developed by inventing and using wheel. Progression of road construction techniques, after the development of the toll system, and especially in the century after 1750, led to the emergence of skilled road builders such as ThomasTelford and John Ludon McAdam (O'Flaherty 2002).

During the past twenty years, developing countries have experienced very huge population growth (Buhaug and Urdal 2013). Urban sprawl and auto mobilization have led to an increase in the number of journeys made by private motorized vehicles, leading to increased congestion, increased greenhouse gas emissions; all this and more resulting in negative environmental, social as well as economic impacts (Sohoni, Thomas and Krishna Rao 2017).

Considering differences between decision-making in goods and private/passenger transport in city administrations (Behrends, Lindholm nad Woxenius 2008), public Transportation is one of the most efficient modes of transport which provide the required mobility (Polat 2012). On a general basis, public transport service suppliers have to study and consider multiple factors to insure the productivity and efficiency of the services (De Borger and Kerstens 2006; Barnum, McNeil and Hart 2007). Public transportation involves regular bus, electric bus, tram and urban railway, high-speed transportation and passenger boats. Suburban public transportation mainly includes taxis, airlines, intercity buses, and intercity railways. Among the various modes of public transport, taxi industry is one of the most heavily regulated industries in the world (Kamga, Yazici and Singhal 2015). Public transportation brings advantages such as high speed, high efficiency, safety, reduction of negative environmental effects, fast implementation of public transportation, stability in value and land use, less traffic congestion, significant volume in passenger transportation, lower costs due to fuel consumption and car depreciation, etc. Tyrinopoulos and Antoniou (2008) aimed to evaluate and understand better passenger attitudes toward public transport modes. With emphasis on the importance of fuzzy logic systems in solving traffic and transportation problems, Sarkar, Sahoo and Sahoo (2012) presented an analysis of the results achieved using fuzzy logic to model complex traffic and transportation processes.

Various strategies can be implemented for improving and developing the transportation system such as supporting the public transportation, increasing the cost of parking cards for private cars, increasing tax on private vehicles and regulating the transportation system by eliminating delays through developing the scenarios (Suryani. et al, 2020). Employing a dynamic system for evaluation of future strategies in Indonesia with considering operational efficiency, service efficiency and uncertainty factors, Suryani et al. (2020) evaluated the effect of simulated models and development of scenarios on improving transportation systems and decreasing traffic congestion.

Debdatta, Subrata and Mitra (2016) measured technical efficiency of road transportation modes in India using Directional Distance Function (DDF) to analyse 
the production function with favourable and unfavourable outputs, and Data Envelopment Analysis (DEA) to measure the technical efficiency of 37 road transportation modes in India. Considering the intelligent systems, as the advanced tools for improving and protecting critical components of infrastructure which can be used for protecting vital road and rail transportation infrastructure components, Janušová and čičmancová (2016) focused on improving transportation safety using intelligent transportation systems.

Discussing the challenge of sustainability for transportation to and from suburban universities, Göçer and Göçer (2018) provided statistical analyses of travel mode choices and their effect on campus use and activity participation for two different demographic groups. Methods of arranging the transportation of passengers in suburban traffic include a large number of system measures that cover both the public transport system and the environment of the system where it exists (Vakulenko et al., 2018). Kubiak and Długosz (2019) proposed a system for optimizing and improving automated and intelligent public transportation systems used in relatively small suburban areas in Poznan, Poland. Such a public transportation transfers passengers from the above-mentioned places to the nearest railway system in the region, from where the departure to other main parts of the city starts by train. Sen et al. (2019) evaluated the reliability of travel time using different public vehicles on a specific route in Calcutta. Cieplińska (2019) discussed the substantial role of transport organizers in overseeing the mechanisms of all flows in the urban area by the advent of automation automation technology. Noting that the COVID-19 pandemic is causing disruptions in the urban transportation system by having detrimental effects on travel and delivery services and the need to review existing transportation issues and provide innovative solutions, Sun et al. (2020) explored a urban-based automated public transportation system (APTS) that serves both people and freight, using emerging technologies such as information technology and automated vehicles.

Proposing the dynamic system model for developing interaction and feedback among private vehicle users, public transport passengers, public transport companies, and the government, Sopha and Ma'mun (2020) evaluated the selection of transportation mechanisms, as well as potential energy policies in the public transportation system. Moore (2019) evaluated the energy-saving potential of innovative means of delivering intra-city freight. Jin and Zhou (2020) investigated the factors affecting the comfort of passengers in the process of transportation and discussed the comfort and convenience of passengers in the public transportation system in the suburbs of Beijing.

The review of literature reveals that the studies related to suburban public transportation based on dynamic simulation are found to be few. In this paper, two simulation models related to the current system and the proposed suburban public transportation system are designed and implemented. An ANFIS is employed for 
route traffic estimation and Fuzzy C-Means (FCM) clustering algorithm is utilized to determine the number of passengers referring to each city terminal, and finally, the Dijkstra's algorithm is applied to find the optimal routing of passengers from the source to the destination.

\section{Preliminaries}

\subsection{Adaptive Neuro-Fuzzy Inference System (ANFIS)}

Fuzzy set theory is a perfect tool to model uncertainties and imprecision arising from mental phenomena. Fuzzy Inference System (FIS) is one of the most popular systems developed for fuzzy reasoning which uses fuzzy logic for modeling uncertainties. ANFIS is a combination of two soft-computing methods of Artificial Neural Networks (ANNs) and fuzzy logic (Jang, 1993). It is an adaptive network that uses supervised learning on learning algorithm, which has a function similar to the model of Takagi-Sugeno FIS (Shokouhifar and Jalali, 2017). Each node in this network has different functions and tasks, and the output depends on the incoming signals and parameters that are available in the node (Suparta and Alhasa, 2016). A learning rule could affect the parameters in the node and reduce the occurrence of errors in the output of the adaptive network (Jang, 1993).

ANFIS integrates neural networks and concepts of fuzzy logic and applies the possibilities of both in one frame, the inference system of which is consistent with the fuzzy if-then set of rules which can be learned for approximating nonlinear functions (Jang, 1993). It is one of the most efficient techniques in the fields of forecasting, estimating, and modelling (Nayak et al, 2004). In the present study, the ANFIS is used to estimate the volume of daily traffic. Fig. 1 shows the general structure of the ANFIS model with two inputs and one output.

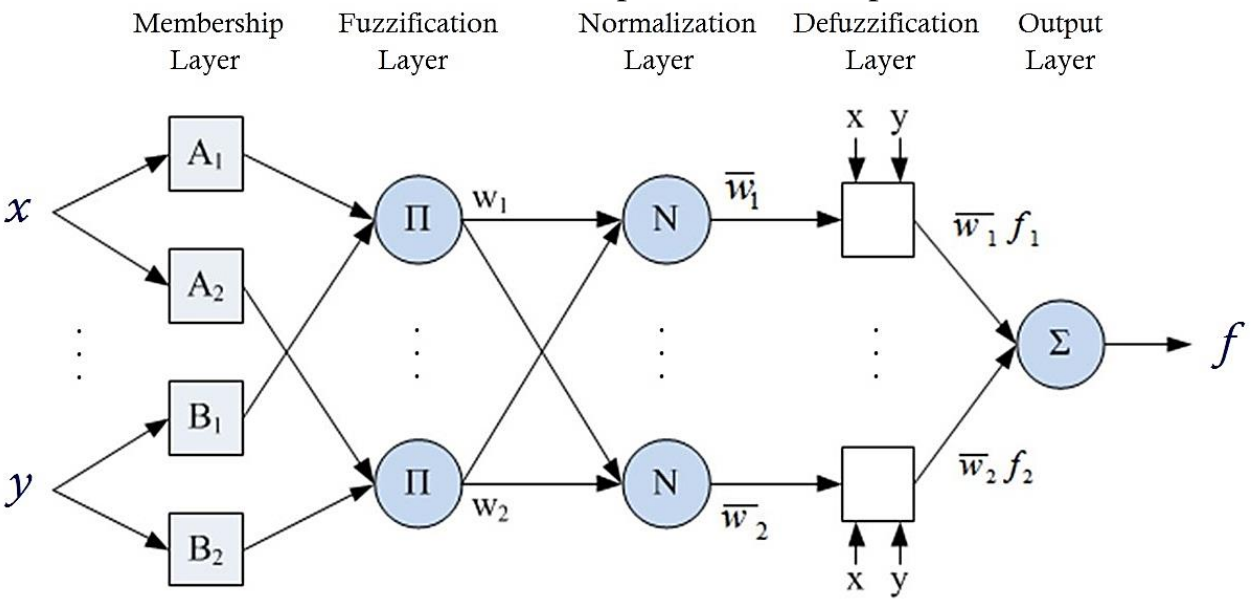

Fig. 1: General structure of ANFIS model with a two-input first-order Sugeno fuzzy model with two rules. 
In the ANFIS model, the fuzzy rules can be expressed as follows:

Rule1: if $\mathrm{x}$ is $\mathrm{A} 1$ and $\mathrm{y}$ is $\mathrm{B} 1$ then $\mathrm{f} 1=\mathrm{p} 1 \mathrm{x}+\mathrm{q} 1 \mathrm{y}+\mathrm{r} 1$;

Rule2: if $\mathrm{x}$ is $\mathrm{A} 2$ and $\mathrm{y}$ is $\mathrm{B} 2$ then $\mathrm{f} 2=\mathrm{p} 2 \mathrm{x}+\mathrm{q} 2 \mathrm{y}+\mathrm{r} 2$;

First layer: In this layer, the membership degree of input nodes to different fuzzy intervals is determined using membership functions. $x$ or $y$ represents the input to node $i$, and $A_{i}$ or $B_{i}$ represent the linguistic variables corresponding to this node.

$$
O_{i}^{1}=\mu_{A_{i}}(x)=\left[1+\left(\left[\frac{x-c_{i}}{a_{i}}\right]^{2}\right)^{b_{i}}\right]^{-1}
$$

Second layer: Each node in this layer is a fixed node called $P$ whose output is the product of all input signals.

$$
O_{i}^{2}=w_{2}=\mu_{A_{i}}(x) \times \mu_{B_{i}}(y) ; \quad i=1,2
$$

Third layer: This layer normalizes the output of the second layer and transfers it to the next layer.

$$
O_{i}^{3}=\overline{w_{i}}=w_{i}^{l} \sum w_{i}
$$

Fourth layer: Each node $\mathrm{i}$ in this layer is a node corresponding to the node function, as follows.

$$
O_{i}^{4}=\overline{w_{i}} \times f
$$

where $\overline{w_{l}}$ represents a normalized firing strength of the third layer, and $p_{i}, q_{i}$, and $r_{i}$ indicate the set of parameters in this node, respectively. The parameters of this layer are called inferential parameters.

Fifth layer: The only node in this layer is a fixed node called, calculating all outputs as a set of all input signals as follows.

$$
O_{i}^{5}=\sum \overline{w_{i}} \times f
$$

\subsection{Dijkstra's Algorithm}

Dijkstra is considered as one of the famous algorithms for calculating the shortest path, developed by the Dutch computer scientist named Edsger W. Dijkstra in 1959 (Dijkstra, 1959). This algorithm is one of the graph navigation algorithms, solving the problem of the shortest path from the single source for the weighted graphs having edges with non-negative weight. Finally, this algorithm obtains the shortest path from the source to all vertices of the graph by creating the shortest path tree. The process of this algorithm for finding the shortest path from the source to the top 
of the destination is such that the algorithm can be stopped during the implementation of the algorithm while finding the shortest path from the source to the destination. If the graph has an edge with negative weight, this algorithm fails to work properly and other algorithms such as the Bellman -Ford algorithm should be used, which are more complex in time. Furthermore, the time order of this algorithm equals $\mathrm{O}(\log (\mathrm{N}) * \mathrm{E})$. The steps of Dijkstra's algorithm are as follows:

The set $\mathrm{S}$ including the graph vertices is determined. At the beginning, this set is empty and this set contains the vertices to which the shortest path is found when the algorithm progresses. The source vertex with the index puts zero inside S. For vertices outside $S$, an index equal to the edge length + the index of the previous vertex is considered. If the vertex outside the set has an index, the new index is the lowest value between the previous index and the edge length + index of the previous vertex. From the vertices outside the set, a vertex with the lowest index is selected and added to the set $\mathrm{S}$. Repeat this until the destination vertex enters the set S. Finally, if the destination vertex has an index, its index indicates the distance between the source and destination. Otherwise, there is no path between the source and the destination (Diestel, 2010).

\section{Proposed ANFIS-Dijkstra's Model}

In the present descriptive and analytical study, library and field methods are used for collecting data. The distance of each city to another city is saved and used in different parts of the program. Based on the latest data, these numbers are extracted one by one through Google Map and new roads are considered. For a number of clients, it is specified how many passengers travel to the terminals of each city by bus or taxi every day and at any minute of the day. Such numbers are adjusted based on the average capacity of buses using random number generation functions to cover the maximum of different situations. The rate of traffic is determined at any given time on the day of the week and minute of the day from each city to other cities. Traffic rate is used as a factor in the simulation. Bus schedule contains 30 excel sheets, specifying the time of buses to different cities and passenger capacity in a separate sheet for each city, which is documented separately for each city using bus departure time information on reputable sites and stored in a single file. For bus prices, the amount of bus fare is mentioned and the information of all cities is stored in one page.

In order to conduct the present study, first the distance between the provincial capitals is stored and the base time for transferring from one city to another is determined based on the average speed of taxis and buses in each route. Route traffic is one of the details considered in this study. Accordingly, the base time for transferring from one city to another is affected. For this purpose, the desired time traffic of the system is estimated by creating an ANFIS model. In order to create the 
desired ANFIS model, three inputs such as the route type, day of the week, and minutes of the day, as well as one output including the traffic coefficient of that route are considered.

To efficiently simulate the transportation system, the number of passengers from each source is estimated using the FCM (Shokouhifar \& Farokhi, 2010) using a field study in relation to the amounts of the different requests at different times to different destinations to be closer to reality. At this stage, other information such as destination, passenger priority including cost or time and the passenger's desired time frame for reaching the destination is received from the passenger, being randomly assigned to the passenger at the same time as defining a new passenger in the system. Then, the study is divided into two parts including the simulation of the current suburban public transportation system and the proposed transportation system.

In the current system simulation, the passenger is answered based on his/her selected priority (cost or time) by submitting a travel request. In the simulation of the proposed system, the passenger is offered the most optimal route based on his request by receiving the passenger's information, which may be performed in combination with taxi and bus during the distance from the source to the destination to be more appropriate for the passenger's request. For this purpose, the Dijkstra's algorithm is used for finding the best route. Finally, the results of simulating the two suburban public transportation systems are compared to determine which could meet the needs of passengers.

\section{Performance Evaluation}

The proposed ANFIS-Dijkstra's model has been successfully developed in MATLAB R2020b. Experiments were carried on a PC with $2.5 \mathrm{GHz}$ i7 processor and 16 GB memory running on windows 10 . In the FIS, Gaussian functions have been employed to define the input and output variables for the fuzzy inference system. The range of variables is defined between 0 and 10. Fig. 2 shows the membership function of the linguistic variables in the FIS.

In the present study, two limitations are considered due to the greater focus on the subject such as restricting vehicles to taxi and bus and restricting the transit between provincial capitals. Therefore, the transportation routes between the provincial centres of Iran are studied for simulating the dynamic suburban public transportation system. Since the traffic on the routes is considered as an effective parameter on the efficiency of public transportation systems, the best diagnosis in this field is diagnosed by creating and training an ANFIS model to lead to a more accurate simulation model. In order to increase the reliability of traffic estimation, the data are divided into three sections of training (70\%), testing (10\%), and checking (20\%). Thus, 140 data out of 200 data are extracted randomly using an appropriate function in MATLAB software and the ANFIS model is taught using such data. 


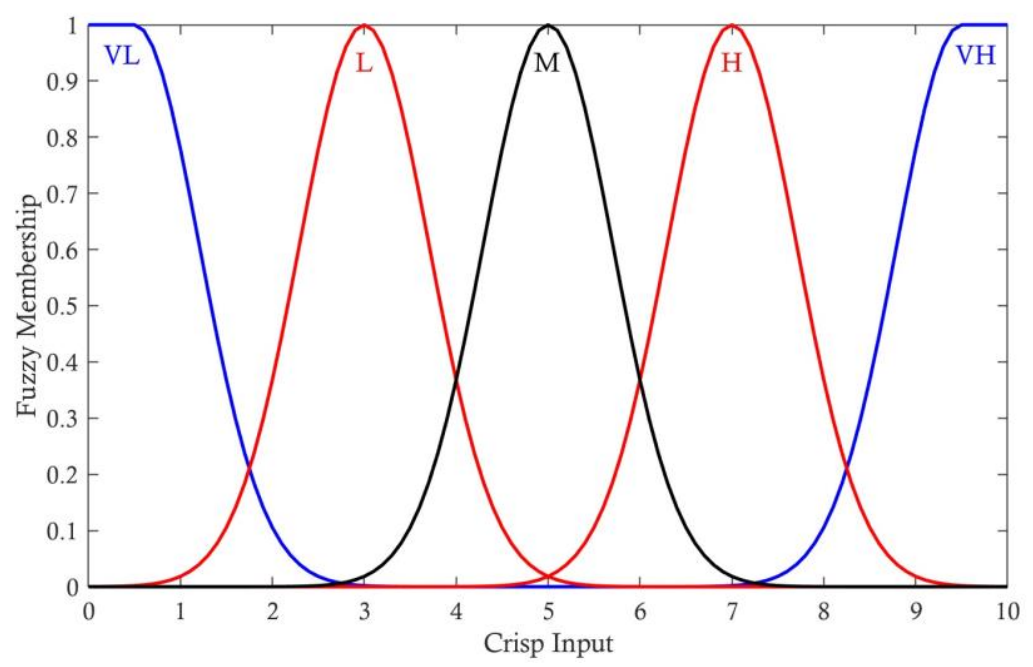

Fig. 2: Membership functions for linguistic variables.

\subsection{Estimating Traffic from Different Routes uing ANFIS}

Fig. 3 displays the training error rate of the ANFIS model generated using the sub clustering method in the training course. As shown, the ANFIS model training has no effect on reducing the model error since the course 140 onwards, indicating that the appropriate selection of number 200 for the number of model training courses using the sub clustering method and the error rate in the training phase is 0.054 . The number of membership functions for inputs is considered 24. Hybrid optimization method is used for training the parameters of membership functions. The used fuzzy inference system is Sugeno with Gaussian membership functions and the variables are converted to fuzzy variables by trial and error using the Gaussian function. The weighted average function is used for defuzzification. After creating the model and its training on the training data, testing and checking data which are not involved in the training process are used for evaluating and determining the validity of the designed model.

Fig. 4 and 5 illustrate the error rates of the generated ANFIS model for the testing and checking datasets, respectively. In these figures, the symbols $\left(^{*}\right)$ indicate the output of the system and the symbol (+) indicates the testing (checking) data generated by the ANFIS model. The calculated mean error rate in testing and checking datasets have been achieved as 0.075 and 0.09 respectively. As a result, no over-fitting is observed in the designed model and the high accuracy of the created model in estimating instantaneous traffic. 


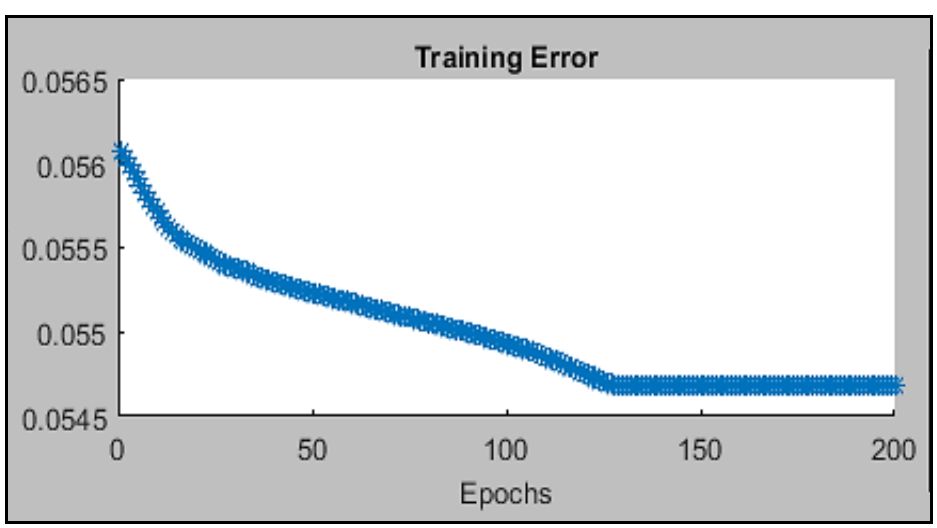

Fig. 3: Training error of the ANFIS model created by the sub-clustering method.

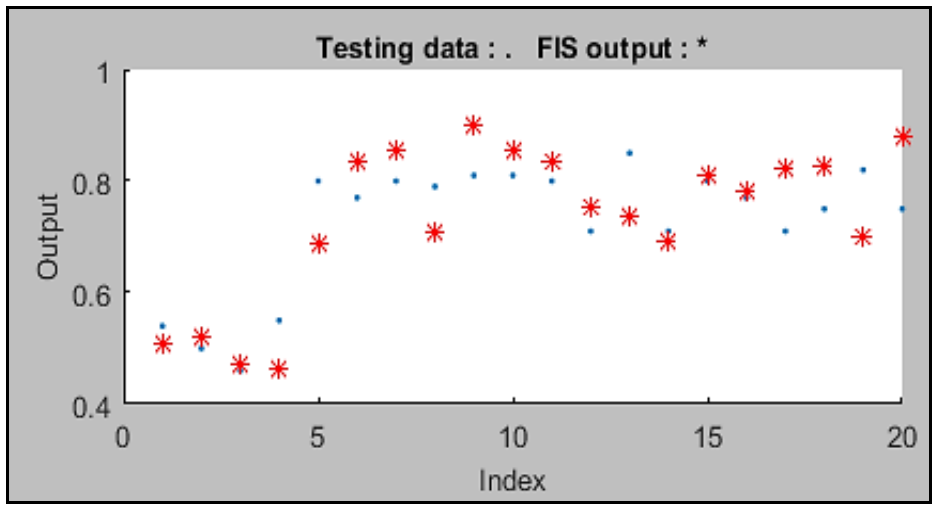

Fig. 4: Testing data error diagram created in the ANFIS model.

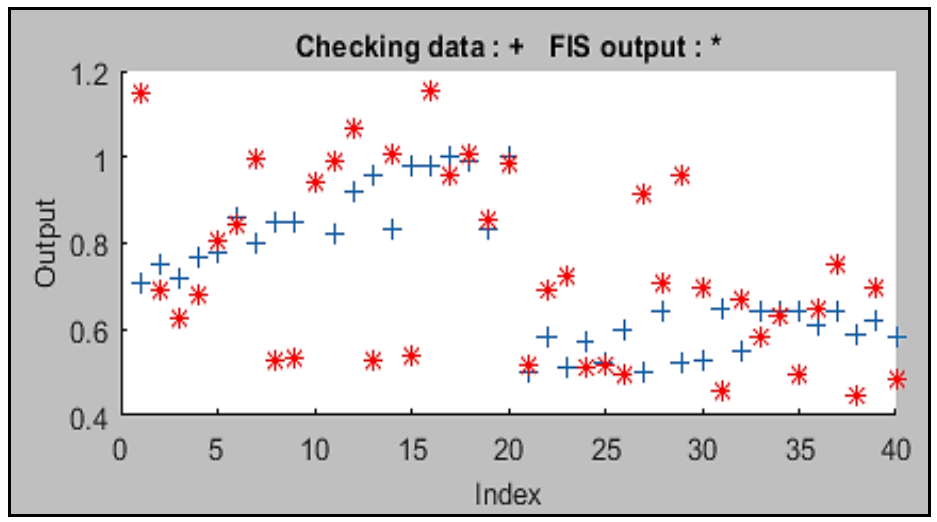

Fig. 5: Checking data error diagram in the generated ANFIS model. 


\subsection{Sensitivity Analysis of the ANFIS Model}

Due to the significance of detecting the traffic rate and its effect on the implementation of the simulation model and ensuring the correct selection of inputs used in creating the ANFIS model, the estimated traffic sensitivity for each input are calculated using the Neuro-Fuzzy-Designer tool as shown in Table 1. As shown in Table 1, the days of week has a higher effect on the performance of the ANFIS traffic estimation model than the other two inputs. In general, using all of the three inputs has a significant effect on reducing the error of the ANFIS model detection. For example, the model error rate is 0.232 without considering the days of week, while this error rate reduced to 0.054 by using this input along with two other variables, indicating a reduction of 0.178 or a $77 \%$ reduction in training error. Based on the numbers obtained from the sensitivity analysis of the inputs used in the ANFIS model, the effect of each input in determining the traffic rate is determined, indicating the appropriate selection of inputs.

Table 1: Effect of each input on the performance of the traffic estimation model.

\begin{tabular}{lccc}
\hline Input & $\begin{array}{c}\text { Error rate } \\
\text { without } \\
\text { calculating input }\end{array}$ & $\begin{array}{c}\text { Error reduction } \\
\text { rate including } \\
\text { input }\end{array}$ & $\begin{array}{c}\text { Percentage of positive } \\
\text { effect of input on error } \\
\text { reduction }\end{array}$ \\
\hline Route type & 0.098 & 0.044 & $45 \%$ \\
\hline Days of week & 0.232 & 0.178 & $77 \%$ \\
\hline Hours of day & 0.116 & 0.062 & $53 \%$ \\
\hline
\end{tabular}

\subsection{Simulation of Current Transportation System}

Fig. 6 displays the current system simulation model. As shown in the upper part of the figure, each pulse of the 120-minute simulation model equals two hours and the related subsystem of all cities is connected to this pulse to update the passenger and bus information at two-hour intervals.

To explain the simulation, the Arak city subsystem is selected as an example: one of the inputs is time that 2 hours per pulse is added to its value. 29 inputs are related to the passengers who had come from different cities to Arak and traveling to another city or their destination is Arak. 29 outputs are connected to other cities, to direct passengers to their next destinations. Another output specified for the Arak subsystem, called Arak Centre, included the passengers who reached their destination in a pulse and their information is sent to the simulation centre for analysis. The three inputs and outputs of the lower part of the subsystem, being connected to each other by a memory block, include:

Q_M: List of passengers waiting for their bus leaving from Arak.

W_M: List of passengers on the road waiting to reach their destination to transfer their information to one of the above-mentioned 29 outputs. 


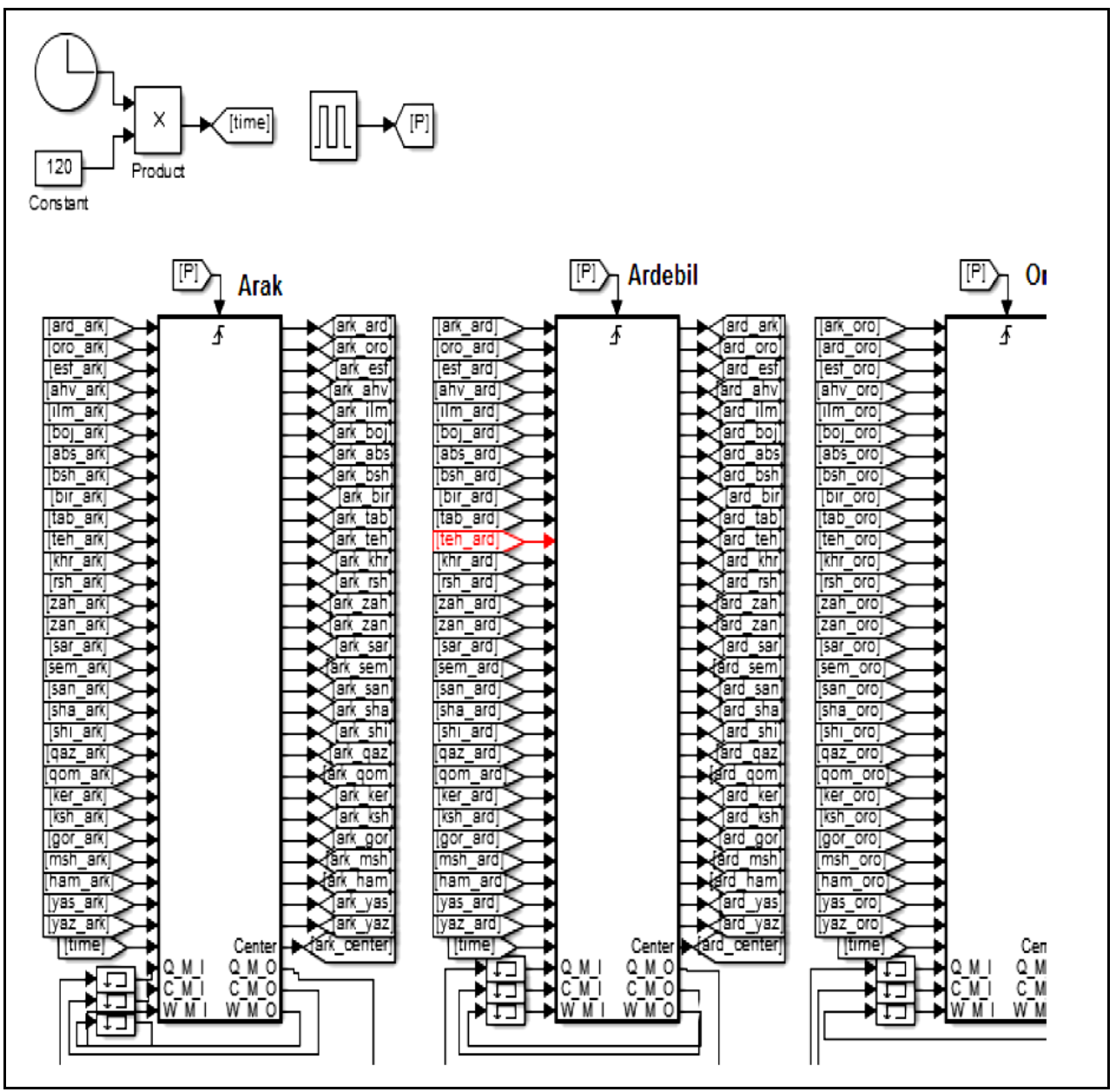

Fig. 6: Part of the simulation model for the current transportation system.

C_M: This list is related to the remaining time and capacity of buses from different origins to different destinations which should be updated on each pulse.

Fig. 7 displays the contents of the Arak city subsystem and its performance is summarized as follows:

Box 1: List of passengers who are on the road from previous pulses.

Box 2: Deciding on new passengers entering the city.

Box 3: Deciding on reaching or not reaching the end of the current route.

Box 4: Preparing the information of the passengers who have not yet arrived at the destination for transfer to the next pulse.

Box 5: Preparing the distance of different routes separately for each city. 


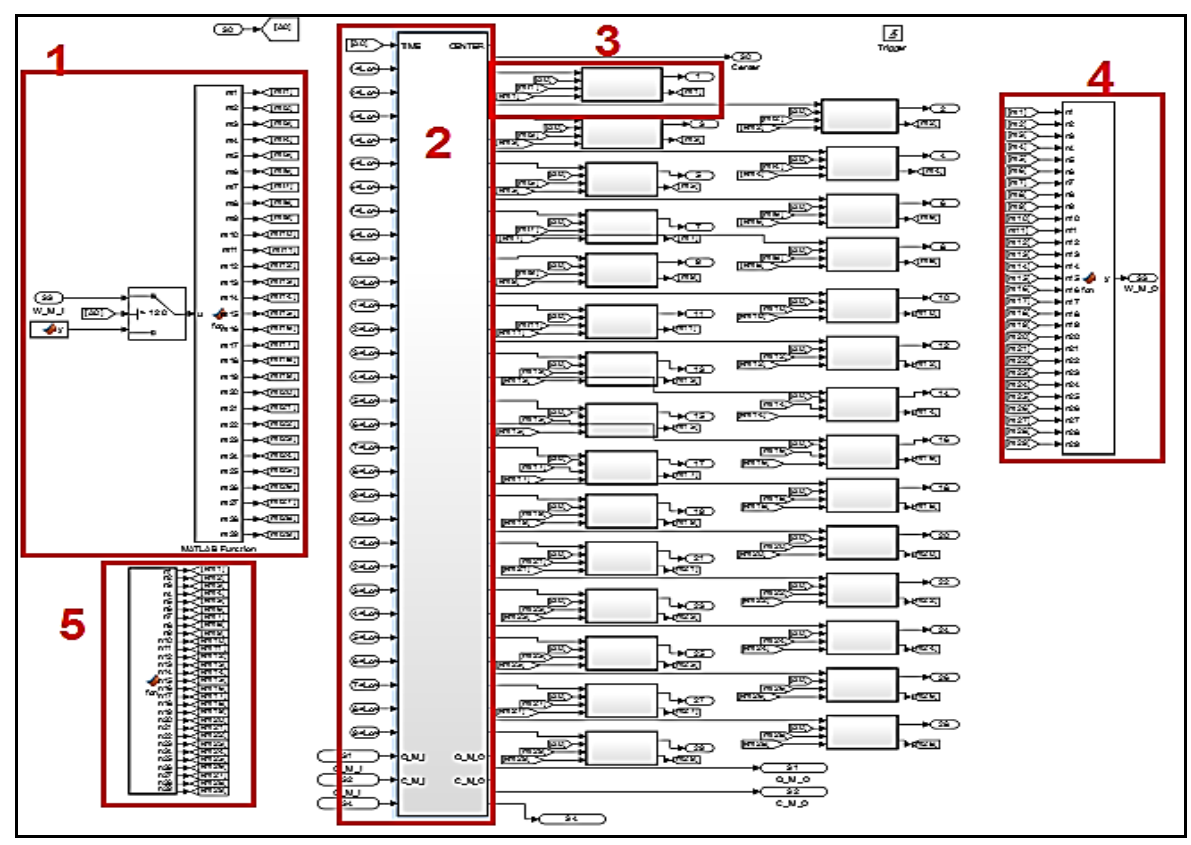

Fig. 7: The contents of the selected subsystem.

Fig. 8 presents the section for analysing and displaying the results of the simulation model. In each time period from each city, the information of all the passengers who have reached their final destination is transferred to this section and the results are calculated:

Box 1: Converting the passengers' information from all cities into a single matrix and directing it to the next subsystem shown in Box 2.

Box 2: This subsystem calculates the three total numbers of passengers, the total paid fare, and the percentage of passengers using bus.

\subsection{Simulation of Proposed Suburban Transportation System}

Fig. 8 displays an overview of the proposed system simulation model. The description of each subsystem in Fig. 9 is as follows:

Box 1: Specifying the number of visitors to the terminal of each city.

Box 2: This section determines the information such as the passengers who have arrived at the destination, the number of passengers who have travelled by bus, and the total number of passengers after separating the vector related to the number of passengers visiting each city in each two-hour period to provide to the next section. In addition, it transfers the instantaneous capacity information of buses and passengers (as C_M_O, P_M_O, respectively) to memory for later time intervals. 


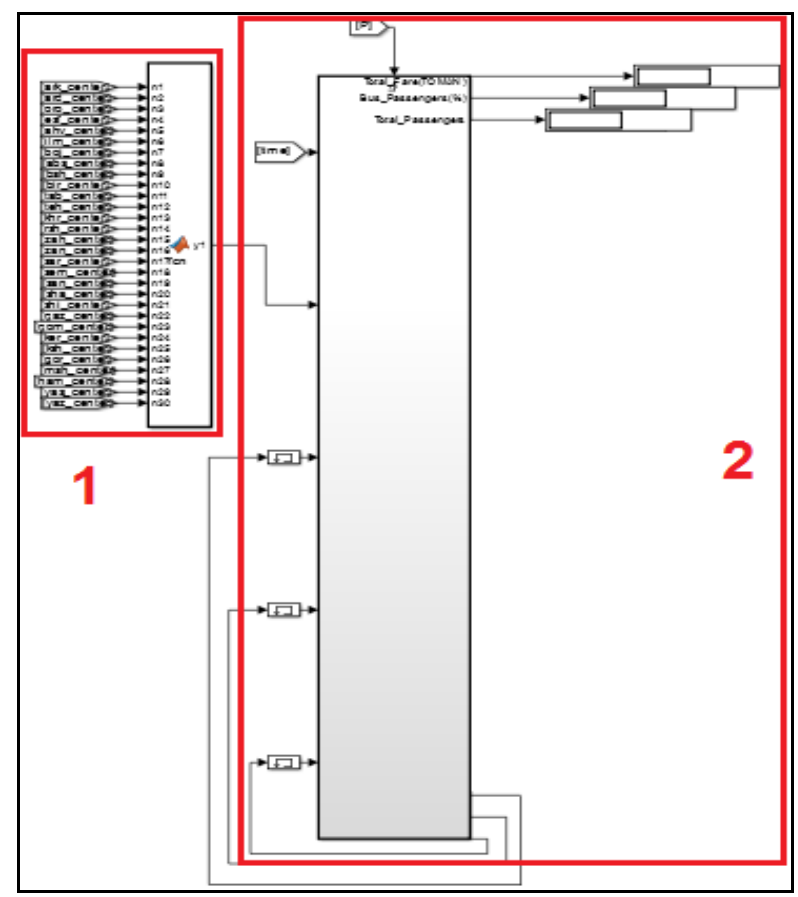

Fig. 8: Section related to analysis and display of simulation model results.

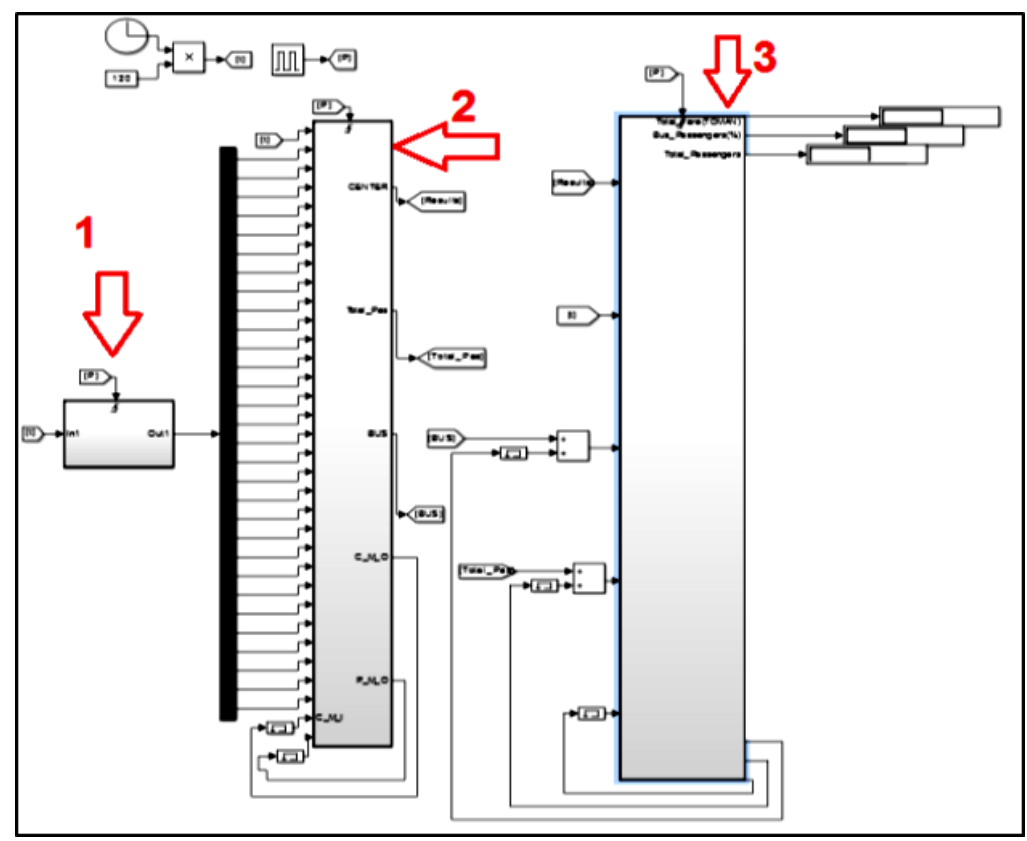

Fig. 9: An overview of the proposed system simulation model.

Moreover, Fig. 10 shows the contents of this sub-system. The description of each subsystem in Fig. 10 is as follows: 
Box 1: In case of being in the first 120 minutes, the passenger information stored in the Passenger file is used; otherwise, the input information to this section being stored in the relevant memory is used.

Box 2: Separating the passengers' information for each city by the name of that city.

Box 3: The information of the passengers whose number is determined in the previous steps using the FCM algorithm is separated and prepared for each city.

Box 4: In this section, the basic information on the capacity of buses or updated capacity is prepared for further use in simulation depending on whether we are in the first 120 minutes or not.

Box 5: In this section, there is a MATLAB function which has the main task in this simulation model.

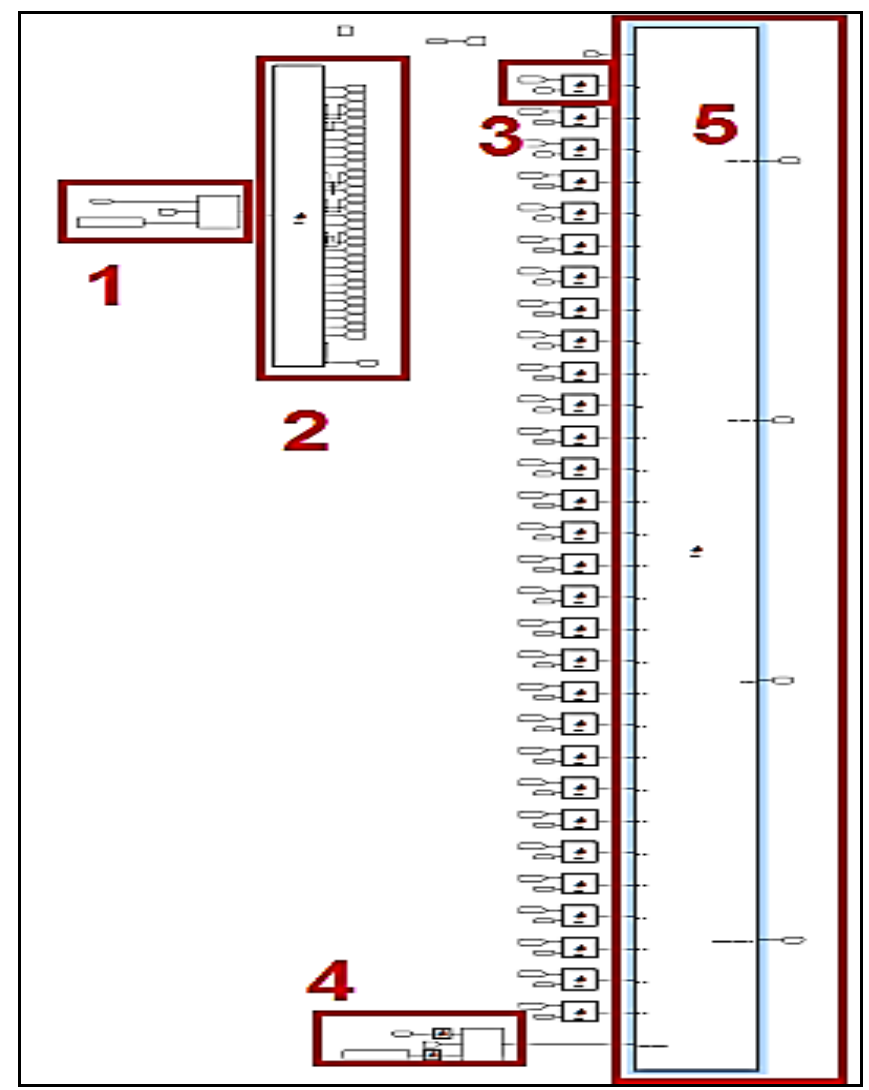

Fig. 10: The contents of the subsystem related to decision-making about passengers in the proposed system.

\subsection{Discussion}

The results of Table 2 are obtained by implementing the simulation models related to the current system and the proposed suburban public transportation system for 
one week. As observed, the use of artificial intelligence algorithms and new methods save a considerable amount of time and money. Obviously, the results of this study indicate their importance when implemented after one or several years. The results of Table 2 along with economic and temporal productivity greatly increase the comfort of passengers in preparing tickets and helping the environment.

Table 2: Comparison of the proposed model with the current system.

\begin{tabular}{lccc}
\hline Input & Current model & Proposed model & Comparison \\
\hline Percentage of using bus & $70.6 \%$ & $78.08 \%$ & $\begin{array}{c}8.2 \% \\
\text { Increase }\end{array}$ \\
\hline Percentage of empty seats & $24.2 \%$ & $11.54 \%$ & $\begin{array}{c}12.66 \% \\
\text { Decrease }\end{array}$ \\
\hline $\begin{array}{l}\text { Average fare paid by each } \\
\text { passenger }\end{array}$ & 818300 & 6432200 & $\begin{array}{c}175100 \\
\text { Decrease }\end{array}$ \\
\hline
\end{tabular}

\section{Conclusion}

In this paper, the studies in the field of public transportation have been reviewed to adopt an appropriate method to optimize fuel consumption, and save cost and time. In the proposed model, an ANFIS model is created for estimating route traffic and the FCM algorithm is established for determining the number of passengers visiting each city terminal. Finally, Dijkstra's algorithm selects the optimal route from the source to the destination. Two simulation models related to the current system and the proposed system of public transportation are designed and implemented for one week while extracting, collecting, and adjusting the data purposefully from the places related to public transportation, and using Simulink and MATLAB software. According to the obtained results, the rate of using buses compared to taxis increased by $8.2 \%$, the maximum capacity of buses increased by $12.66 \%$ and the total fare paid by passengers decreased by 175100 Rials.

The designed transportation system is not limited to optimizing fuel consumption and reducing passenger costs. Based on the maximum use of bus capacity, such a system provides a healthier environment. If we consider the fare paid by the passenger, the passenger's confidence in preparing the ticket and the optimal routing in terms of time among the concerns and components affecting passenger satisfaction, the raised issues will meet all these components. The proposed methodology can be applied to other datasets. The dynamics of the methods used in this study is considered as one of the reasons. Another reason is that decisionmaking models adapt themselves to training data using artificial intelligence. As a future work, other machine learning techniques such as Co-Active Neuro-Fuzzy Inference System (CANFIS) can be utilized and evaluated for suburban public transportation. 


\section{References}

Barnum, D. T., McNeil S. and J. Hart (2007). Comparing the efficiency of public transportation subunits using data envelopment analysis. Journal of Public Transport, 10, 116.

Behrends, S., Lindholm M. and J. Woxenius (2008). The Impact of Urban Freight Transport: A Definition of Sustainability from an Actor's Perspective. Transportation Planning and Technology, 31(6), 693-713.

Buhaug, H. and H. Urdal (2013). An urbanization bomb? Population growth and social disorder in cities. Global Environmental Change, 23(1), 1-10.

Cieplińska, J. R. (2019). The Role of Transport Organisers in the Integration of Passengers and Goods Flows Within Urban Areas. Transportation Research Procedia, 39, 453-461.

De Borger, B. and K. Kerstens (2006). The performance of bus transit operators. Document de travail du LEM. 20063 (21).

Debdatta, P., Subrata K. and A. Mitra (2016). An application of the directional distance function with the number of accidents as an undesirable output to measure the technical efficiency of state road transport in India. Transportation Research Part A: Policy and Practice, 93, 1-12.

Diestel, R. (2010). Graph Theory. 4th Edition, springer, Berlin, Heidelberg.

Dijkstra, E.W. (1959). A note on two problems in connexion with graphs, Numerische mathematic, 1, 269-271.

Gill, J. and J. Singh (2017). Performance analysis of vapor compression refrigeration system using an adaptive neuro-fuzzy inference system. Int. J. Refrig. $82,436-446$.

Göçer, O. and K. Göçer (2018). The Effects of Transportation Modes on Campus Use: A Case Study of a Suburban Campus. Case Studies on Transport Policy.

Hidalgo, D. and C. Huizenga (2013). Implementation of sustainable urban transport in Latin America. Research in Transportation Economics, 40(1), 66-77.

Hutchison, R. (2010). Encyclopedia of urban studies. SAGE Publications. ISBN (Electronic): 9781412971973. 
Jang, J. S. R. (1993). ANFIS: Adaptive Network Based Fuzzy Inference System. IEEE Transactions on Systems, Man and Cybernetics, 23(3), 665 - 685.

Janušová, L. and S. Čičmancová (2016). Improving Safety of Transportation by Using Intelligent Transport Systems. Procedia Engineering, 134,14-22.

Jin, T. and Y. Zhou (2020). Research on the Development of Beijing Suburban Public Transportation Based on SEM. Journal of Physics, 1549(2), 022-027.

Kamga, C., Yazici M.A. and A. Singhal (2015). Analysis of taxi demand and supply in New York City: implications of recent taxi regulations. Transportation Planning and Technology, 38(6), 601-625.

Kubiak, M. B. K. and R. Długosz (2019). Solutions for Planning Smart Hybrid Public Transportation System-Poznan Agglomeration as a Case Study of Satellite Towns' Connections. Communication Papers of the Federated Conference on Computer Science and Information Systems, 20, 67-72.

Li, Y. and M. DaCosta (2013). Transportation and income inequality in China: 1978- 2007. Transportation Research Policy and Practice, 55, 56-71.

Moore, A.M. (2019). Innovative scenarios for modeling intra-city freight delivery. Transportation Research Interdisciplinary Perspectives, 3, 100024.

Nayak, P. C., Sudheer K. P., Rangan D. M. and K. S. Ramasastri (2004). A NeuroFuzzy Computing Technique for Modelling Hydrological Time Serie. Journal of Hydrology, 291(2), 52-66.

O'Flaherty, C.A. (2002). Introduction: A historical overview of the development of the road, Highways (Fourth Edition), Butterworth-Heinemann, 1-5.

Polat, C. (2012). The Demand Determinants for Urban Public Transport Services: A Review of the Literature. Journal of Applied Sciences, 12(12), 1211-1231.

Sabet, S., Farokhi, F., and Shokouhifar, M. (2013). A hybrid mutation-based artificial bee colony for traveling salesman problem. In 4th International Conference on Electronics Computer Technology (ICECT).

Sarkar, A., Sahoo G. and U. C. Sahoo (2012). Application of fuzzy logic in transport planning. International Journal on Soft Computing 3(2). 
Sen, S., Chowdhury T., Mitra A. and S. K. Roy (2019). Assessing Travel Time Reliability of Public Transport in Kolkata: A Case Study. In Advances in Transportation Engineering: 21-34, Springer, Singapore.

Shokouhifar, M., and Farokhi, F. (2010). Feature selection using supervised fuzzy C-means algorithm with ant colony optimization. In 3rd International Conference on Machine Vision (ICMV), 441-446.

Shokouhifar, M., and Jalali, A. (2015). A new evolutionary based application specific routing protocol for clustered wireless sensor networks. AEU-International Journal of Electronics and Communications, 69(1), 432-441.

Shokouhifar, M., and Jalali, A. (2017). Optimized sugeno fuzzy clustering algorithm for wireless sensor networks. Engineering applications of artificial intelligence, 60, 16-25.

Shokouhifar, M., and Jalali, A. (2017). Simplified symbolic transfer function factorization using combined artificial bee colony and simulated annealing. Applied Soft Computing, 55, 436-451.

Shokouhifar, M., Sabbaghi, M. M., and Pilevari, N. (2021). Inventory Management in Blood Supply Chain Considering Fuzzy Supply/Demand Uncertainties and Lateral Transshipment. Transfusion and Apheresis Science, 103103.

Sohoni, A.V., Thomas M. and K. V. Krishna Rao 2017. Application of the concept of transit-oriented development to a suburban neighborhood. Transportation Research Procedia, 25, 3220-3232.

Sopha, B.M. and S. Ma'mun 2020. System dynamics simulation of private and public transportation in a developing country. In AIP Conference Proceedings, 2223(1), 050009, AIP Publishing LLC.

Sun, S., Wong Y.D., Liu X. and A. Rau 2020. Exploration of an integrated automated public transportation system. Transportation Research Interdisciplinary Perspectives, 8, 100275.

Suryani, E., Hendrawan R. A., Adipraja P. F., Wibisono A., Widodo B. and R. Indraswari 2020. Modelling and simulation of transportation system effectiveness to reduce traffic congestion: a system dynamics framework. Transportation Planning and Technology, 43(7), 670-697. 
Tyrinopoulos, Y. and C. Antoniou 2008. Public transit user satisfaction: Variability and policy implications. Transp. Policy, 15(4), 260-272.

Vakulenko, K., Kuhtin K., Afanasieva I. and A. Galkin 2018. Designing Optimal Public Bus Route Networks in a Suburban Area. Transportation Research Procedia, 39, 554-564.

World Energy Outlook (2010). https://www.iea.org/reports/world-energy-outlook$\underline{2010}$.

World Health Organization (2004), World Health Day: Road safety is no accident! https://www.who.int/mediacentre/news/releases/2004/pr24/en/ 